\title{
Vestibular-Evoked Myogenic Potentials as a Test of Otolith Function
}

\author{
Khalid Al-Sebeiha Anthony Zeitouni ${ }^{b}$ \\ aDepartment of Surgery, Faculty of Medicine, Kuwait University, and Department of Otolaryngology, Sabah \\ Hospital, Kuwait; ${ }^{\text {b}}$ Department of Otolaryngology, Royal Victoria Hospital, McGill University, Montreal, Quebec, \\ Canada
}

\author{
Key Words \\ Otolith - Sensorineural hearing loss · Vertigo . \\ Vestibular-evoked myogenic potential
}

\begin{abstract}
Objectives: To evaluate the use of 75 auditory clicks rather than the usual 100-256 for production of the vestibular-evoked myogenic potential (VEMP) response and to assess if the VEMP correlates with measures of cochlear function such as hearing loss (decibels on pure-tone testing) or auditory brainstem response (ABR). Subjects and Methods: Testing of the VEMP was carried out on 24 subjects: 7 normal individuals and 13 hard-of-hearing patients, 2 patients with known vestibular lesions, 1 with profound sensorineural hearing loss and 1 patient with unilateral acoustic neuroma prior to resection. The recording was performed using a conventional ABR machine. Using 75 refraction clicks as auditory stimuli, VEMP responses were measured from a surface elec-
\end{abstract}

Presented at the 52nd Annual Meeting of the Canadian Society of Otolaryngology, Head and Neck Surgery, Montreal, Quebec, Canada, J une 14-17, 1998, and at the Annual Meeting of the Association of Otorhinolaryngology and Maxillofacial Surgery of Quebec, Sainte-Adèle, September 24-27, 1998 (second prize).

\begin{tabular}{ll}
\hline KARGER & ( ) 2002 S. Karger AG, Basel \\
1011-7571/02/0113-0136\$18.50/0 \\
$\begin{array}{l}\text { Fax +4161306 1234 } \\
\begin{array}{l}\text { E-Mail karger@karger.ch } \\
\text { www.karger.com }\end{array}\end{array}$ & $\begin{array}{l}\text { Accessible online at: } \\
\text { www.karger.com/journals/mpp }\end{array}$
\end{tabular}

trode applied to the sternocleidomastoid muscle. The 75 responses were averaged. Results: A consistent unilateral biphasic response, the first negative and the second positive ( $\mathrm{nl}$, pll), was elicited from 39 of 40 ears ( 7 normal and 13 hard-of-hearing subjects with no known vestibular dysfunction). No statistical difference was found between the right and left ear responses for $\mathrm{nl}$ or pll wave latencies. Two patients with loss of peripheral vestibular function showed no VEMP responses on the affected side. The responses, however, were elicited bilaterally in a patient with congenital profound sensorineural hearing loss. In addition, ABR latencies did not correlate with VEMP latencies. Conclusion: Clear VEMP responses were elicited by using only 75 auditory clicks. This shortens the testing time and facilitates the procedure for the tested subject. Our data also indicate that the VEMP is generated secondary to auditory stimulation of the vestibular end organs (specifically the saccule) and does not correlate with cochlear function.

Copyright $\odot 2002$ S. Karger AG, Basel

\section{Introduction}

The vestibular labyrinth is composed of two functionally and anatomically distinct parts, the semicircular canals, which sense angular acceleration, and the otolith organs, which sense linear acceleration and gravity. Con- 
siderable progress has been made in understanding the function and dysfunction of the semicircular canals. This has been facilitated by the availability of semicircular canal tests such as caloric and rotational testing. On the other hand, the ability to diagnose different otolithic dysfunction has been limited by the lack of clinical tests.

Several studies have shown that auditory stimuli induce a response from various muscles, such as the sternocleidomastoid $[1,2]$. These responses, most likely originating in the saccule, have been called vestibular-evoked myogenic potentials (VEMPs) [1,2].

In a series of experiments, Cazals et al. [3] found that, after a total ablation of both the cochlear and the vestibular parts of the inner ear by ototoxic drugs in guinea pigs, no vestibular-evoked myogenic response could be elicited by acoustic stimuli. Using amikacin at doses that destroyed the cochlea but spared the saccule, short-latency responses to acoustic stimuli could be recorded from the round window [4]. Later, Didier and Cazals [5], using microelectrodes, demonstrated that these short-latency responses could be recorded from saccular fibers of the eighth nerve in guinea pigs. McCue and Guinan [6] conducted labeling experiments on cats in an attempt to confirm the saccular origin of these potentials. Using microelectrodes, these authors identified afferent eighth-nerve fibers with irregular spontaneous discharges to loud auditory clicks. The potentials, recorded from these fibers, had the characteristics of a high threshold and short latency, which made them easily distinguishable from the cochlear afferent potentials. These fibers were labeled by injecting biocytin intracellularly. In this way, these researchers were able to establish the presence of bipolar ganglion cells, with cell bodies in the inferior division of the vestibular nerve and peripheral processes projecting through the saccular nerve. Murofushi et al. [7] tested fibers from the inferior division and the posterior part of the superior division of the vestibular nerves of guinea pigs, showing that fibers responsive to static tilt (otolith function) were also responsive to auditory stimuli. McCue and Guinan [8] tested afferent fibers in the inferior vestibular nerve in cats, innervating the saccule and the posterior semicircular canal, and found that a substantial number of these fibers with irregular background activity increased their firing rate in response to auditory clicks. In addition, these responses increased also monotonically with the sound level of the clicks.

Colebatch et al. [1] successfully recorded EMG activity from the sternocleidomastoid muscle in humans in response to acoustic stimuli. This response was biphasic with a mean latency of $13.3 \mathrm{~ms}$ for the first positive wave and $22.6 \mathrm{~ms}$ for the first negative wave. In addition, they showed that these responses were preserved in patients with severe sensorineural hearing loss although they were abolished in patients with complete loss of vestibular function following vestibular neurectomy. Similar results were obtained by substituting auditory clicks with head tapping with a reflex hammer, which apparently induced a sudden acceleration stimulus to the otolith [9]. The responses were invariably absent on the side of the sectioned vestibular nerve [10].

Halmagyi and Colebatch [10] studied 28 patients with unilateral absence of caloric response. Six of these patients had a complete vestibular neurectomy, while others were postvestibular neurolabyrinthitis cases. These authors noticed that no VEMP could be elicited in all patients with vestibular neurectomy. On the other hand, the VEMP was preserved in several patients after vestibular neurolabyrinthitis [10, 11]. Robertson and Ireland [2] found that there was a high directional correlation between p14-n21 potentials and the gold standard caloric testing. In their series, only $75 \%$ of the subjects demonstrated a VEMP response.

The optimal number of clicks needed to acquire a good VEMP response is still controversial. While Colebatch and Halmagyi [1, 12] and Robertson and Ireland [2] used 256 clicks (delivered in two series of 128) at an intensity equal to $100 \mathrm{~dB}$ HL, Bath et al. [13] used 128 clicks of 97 $\mathrm{dB}$ HL, and $\mathrm{Wu}$ and Murofushi [14] used 100 clicks at an intensity equal $95 \mathrm{~dB}$ HL. A study by Robertson and Ireland [2] found that only $75 \%$ of normal subjects had VEMPs. Hence the objectives of this study are to assess the use of 75 clicks rather than the usual number of clicks (100-256) in the production of VEMP responses and to evaluate if VEMP responses correlate with measures of cochlear function, such as hearing loss ( $\mathrm{dB}$ on pure tone testing) or auditory brainstem response (ABR) results.

\section{Subjects and Methods}

\section{Subjects}

Prior to testing, approval was obtained from the Ethics Committee at the Royal Victoria Hospital, McGill University, Montreal, Canada. A group of 7 normal subjects (aged from 25 to 55 years) and 13 hard-of-hearing patients (aged from 40 to 81 years) were tested for VEMP responses using 75 high-intensity rarefaction clicks. The hard-of-hearing patients had undergone pure-tone audiograms with a normal ABR but had hearing loss at $4,000 \mathrm{~Hz}$ ranging from 30 to $80 \mathrm{~dB}$. In addition, testing was conducted on 2 patients with known vestibular lesions, 1 with profound congenital sensorineural hearing loss and the remaining patient with unilateral acoustic neuroma prior to resection. 


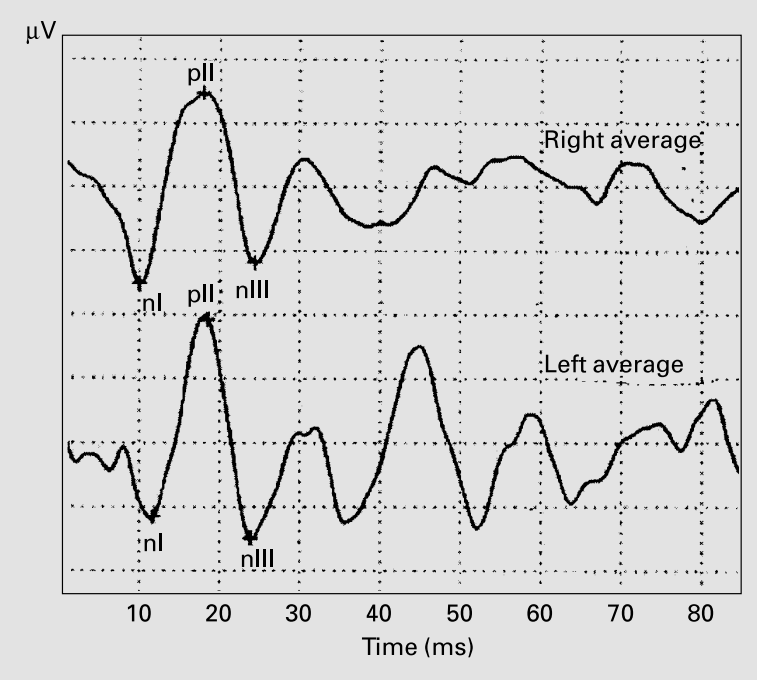

Fig. 1. The VEMP response in a normal subject; note the biphasic responses bilaterally.

\section{Preparation and Testing Procedure}

The recording was performed using a conventional machine (Cadwell Sierra LT with 2-channel averaging capacity, Cadwell Laboratories Inc.). A total of 3 surface electrodes were used: one attached to the upper half of the sternocleidomastoid muscle; the second placed on the ipsilateral mastoid bone (reference electrode); the third placed on the contralateral wrist (ground electrode). The skin overlying these areas was scrubbed and prepared with alcohol in the usual manner. During testing, the subject was in a sitting position and was asked to contract his sternocleidomastoid muscles by pressing the chin against an adjustable Mayo stand.

The 75 high-intensity rarefaction clicks $(100 \mathrm{~dB}$ hearing level, $0.1 \mathrm{~ms}$ duration) were produced by a conventional ABR generator and presented at a rate of 5 per second. EMG activity was recorded from the sternocleidomastoid muscle ipsilateral to the test ear. These recordings were amplified and band-bass filtered from $10 \mathrm{~Hz}$ to $3 \mathrm{kHz}$. A total of 75 clicks were used in cycles of 25 clicks with a break of 1 min between each cycle. The 75 click responses were averaged. Statistical comparisons of VEMP responses were conducted using the nonparametric Mann-Whitney U test. The analysis was performed with the SPSS 9.0 program.

\section{Results}

All 14 ears of the 7 normal subjects showed a VEMP response to the acoustic stimuli. Responses were elicited in 25 out of 26 ears (96\%) in the hard-of-hearing group. The response was biphasic with a first negative (nI) wave followed by a positive wave (pII; fig. 1). For the 39 ears
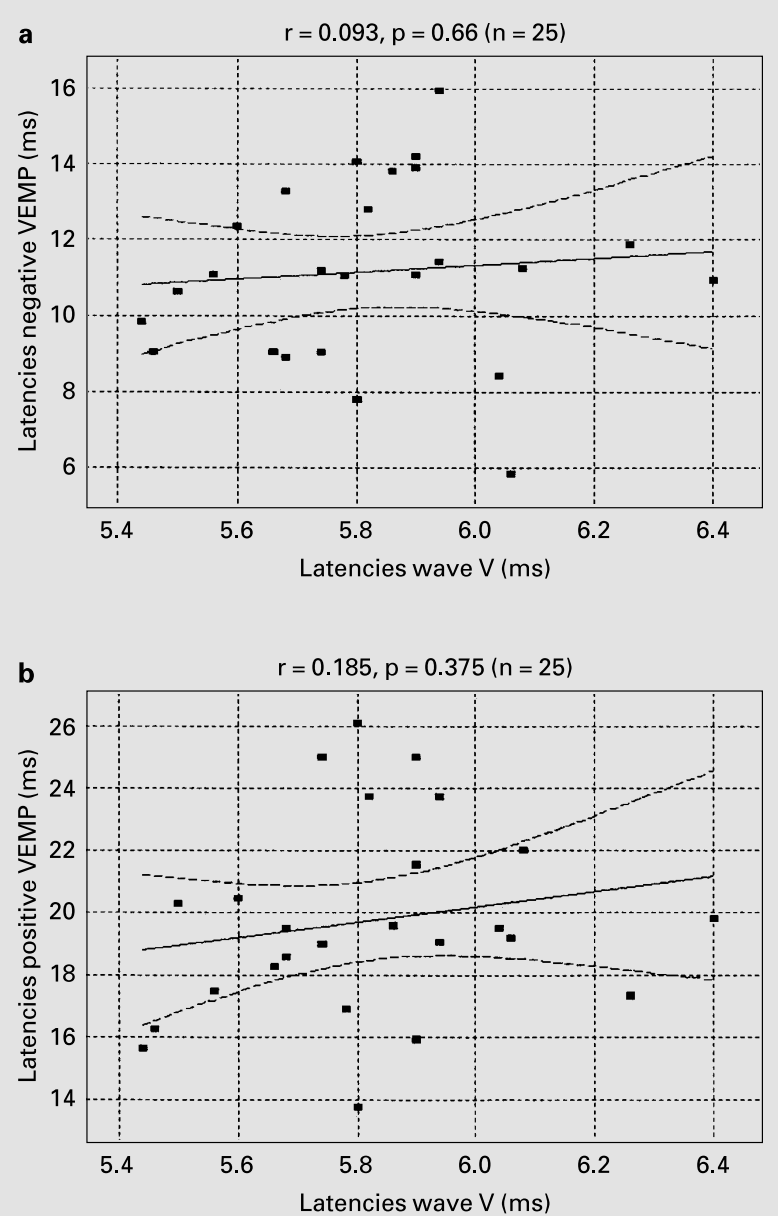

Fig. 2a, b. Wave V latency of the ABR compared to the negative wave latency (a) and positive wave latency (b) of the VEMP.

with the VEMP response, the mean latency for the negative wave $(\mathrm{nI})$ was $11.06 \pm 1.97 \mathrm{~ms}$ (ranging from 5.84 to $15.94 \mathrm{~ms}$ ). The mean latency of the positive wave (pII) was $19.15 \pm 2.68 \mathrm{~ms}$ ranging from 13.75 to $26.09 \mathrm{~ms}$. The latency difference between the $\mathrm{nI}$ and $\mathrm{pII}$ waves ranged from 3.75 to $13.38 \mathrm{~ms}$, with a mean of $8.08 \pm 2.27 \mathrm{~ms}$.

In 19 subjects ( 7 normal and 12 hard-of-hearing), the responses were recorded bilaterally. When left ear responses were compared to right ear responses, there was no statistical difference between the two sides for the latency of either the $n I$ wave $(p=0.92)$ or the $\mathrm{pII}$ wave $(\mathrm{p}=$ 0.72 ). Furthermore, there was no difference in the latency between negative and positive waves $(\mathrm{p}=0.92$; table 1$)$. To compare the latency of wave $V$ of the ABR to the nega- 


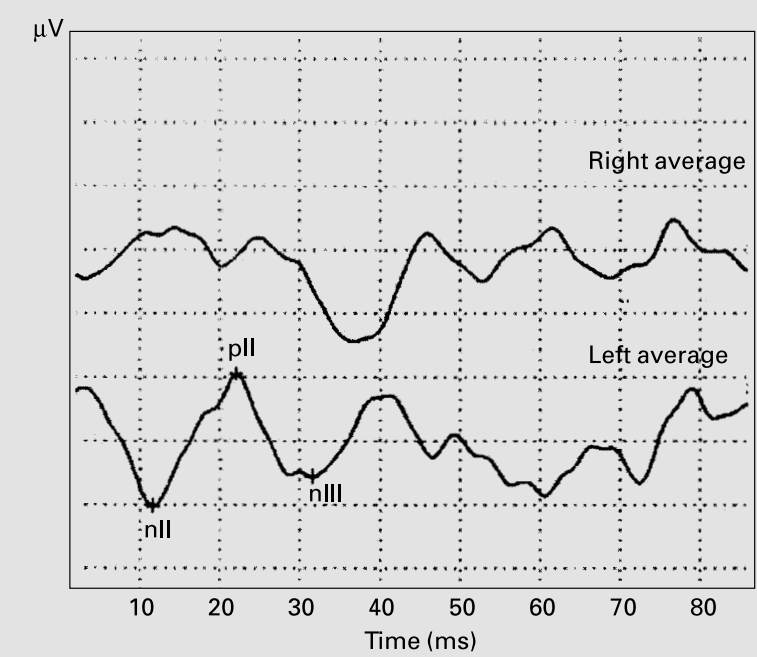

Fig. 3. VEMP responses in a patient with total loss of the left vestibular function following left vestibular nerve section. Note that the VEMP is absent on the affected side.

tive and positive wave latencies of the VEMP, a statistical analysis was done on 25 ears of the hard-of-hearing patients with a normal ABR. The comparison showed no correlation between wave V latency of the ABR and the negative wave $(r=0.093)$ or the positive wave $(r=0.185)$ latencies of the VEMP (fig. 2a, b). Likewise, no correlation was seen between hearing loss (in decibels on puretone testing at $4,000 \mathrm{~Hz})$ and wave $\mathrm{nI}(\mathrm{r}=0.12)$ or $\mathrm{pII}(\mathrm{r}=$ 0.19 ), indicating the noncochlear origin of the VEMP responses.

Of the 4 remaining patients, 2 with known vestibular lesions did not have any VEMP response (fig. 3), while the third with a congenital hearing loss had a VEMP response (fig. 4). In the fourth patients with unilateral acoustic neuroma prior to resection, the VEMP response was preserved on the normal side, while it was lost on the side with the lesion, probably due to pressure from the lesion on the vestibular nerve.

\section{Discussion}

These data suggest that high-intensity clicks elicit VEMP responses from the neck muscles which were detectable in 39 out of 40 ears $(97.5 \%)$. The biphasic response consisted first of a negative wave (nI) and a sec-

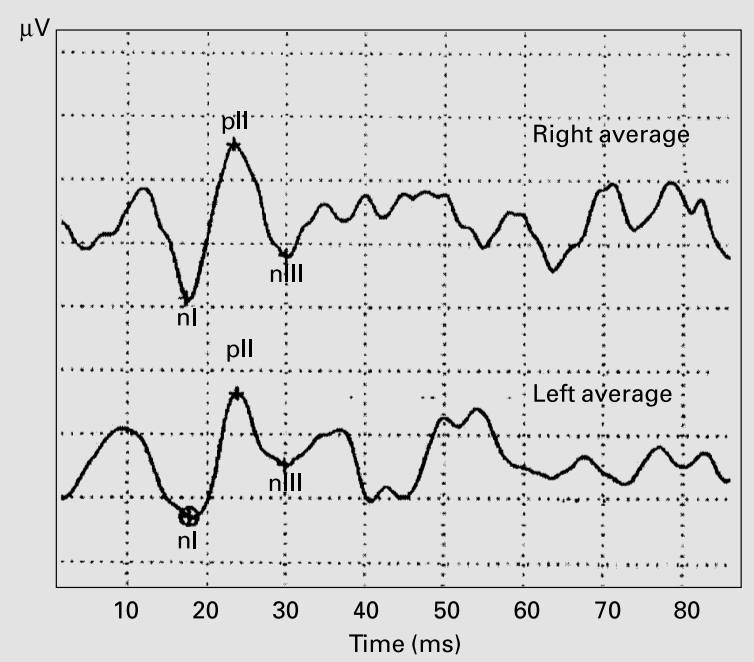

Fig. 4. The presence of VEMP bilaterally in a patient with profound congenital sensorineural hearing loss.

Table 1. The mean latency values (ms) of nI, pII and nI-pII in 19 patients

\begin{tabular}{lrrr}
\hline Peak wave & Right side & Left side & p value \\
\hline nI & $10.94 \pm 1.98$ & $11.18 \pm 2.00$ & 0.92 \\
pII & $19.30 \pm 2.88$ & $19.01 \pm 2.55$ & 0.72 \\
nI-pII & $8.28 \pm 2.61$ & $7.88 \pm 1.89$ & 0.92 \\
\hline
\end{tabular}

ond positive wave (pII), followed by several inconsistent components (nIII, pi). The results obtained in this study are comparable to previous work in terms of the success rate in detecting VEMP responses [1,2]. Whereas other studies have used between 100 and 256 clicks per ear to average the responses, responses in this study were obtained with only 75 clicks delivered in 3 cycles of 25 clicks. This method shortens the time of testing and, more importantly, is more comfortable for the patient who can then push more consistently. Clearly this is a preliminary result that will require further verification and comparison with other click repetition rates. The current study supports the view that VEMP responses are a common finding in normal ears as has previously been demonstrated by Colebatch et al. [1] in 10 normals. 
Most interesting and controversial remains the origin of the VEMP. In this study, we compared the VEMP response to two measures of cochlear function (pure-tone threshold and ABR): no correlation was found between these measures. This would further support the hypothesis that the VEMP is not of cochlear origin [10-12].

\section{Conclusion}

Clear VEMP responses were elicited by using only 75 auditory clicks instead of the usual 256 . This shortens the testing time and facilitates the procedure for the tested subject. Our data suggest that the VEMP is generated secondary to auditory stimulation of the vestibular end organs and does not correlate with cochlear function.

\section{References}

1 Colebatch JG, Halmagyi GM, Skuse NF: Myogenic potentials generated by a click-evoked vestibulocollic reflex. J Neurol Neurosurg Psychiatry 1994;57:190-197.

2 Robertson D, Ireland D: Vestibular evoked myogenic potentials. J Otolaryngol 1995;24: 3-8.

3 Cazals Y, Aran JM, Erre JP, Guilhaume A, Aurousseau C: Vestibular acoustic reception in the guinea pig: A saccular function? Acta Otolaryngol 1983;95:211-217.

4 Cazals Y, Guilhaume A: Otoconia and neural junctions of type I hair cells in amikacintreated guinea pigs presenting saccular acoustic responses. Arch Otorhinolaryngol 1985;242: 155-160.

5 Didier A, Cazals Y: Acoustic responses recorded from the saccular bundle on the eighth nerve of the guinea pig. Hear Res 1989;37:123-128.
6 McCue MP, Guinan JJ: Sound-evoked activity in primary afferent neurons of a mammalian vestibular system. Am J Otol 1997;18:355360.

7 Murofushi T, Curthoys IS, Topple AN, Colebatch J, Halmagyi G: Responses of guinea pig primary vestibular neurons to clicks. Exp Brain Res 1995;103:174-178.

8 McCue MP, Guinan JJ: Acoustically responsive fibers in the vestibular nerve of the cat. $\mathrm{J}$ Neurosci 1994;14:6058-6070.

9 Halmagyi GM, Yavor RA, Colebatch JG: Tapping the head activates the vestibular system: A new use for the clinical reflex hammer. Neurology 1995;45:1927-1929.

10 Halmagyi GM, Colebatch JG: Vestibular evoked myogenic potentials in the sternomastoid muscle are not of lateral canal origin. Acta Otolaryngol 1995;suppl 520:1-3.
11 Murofushi T, Halmagyi GM, Yavor RA, Colebatch JG: Absent vestibular evoked myogenic potentials in vestibular neurolabyrinthitis. Arch Otolaryngol Head Neck Surg 1996;122: 845-848.

12 Colebatch JG, Halmagyi GM: Vestibular evoked potential in human neck muscles before and after unilateral vestibular deafferentation. Neurology 1992;42:1635-1636.

13 Bath A, Harris N, Yardley M: The vestibulocollic reflex. Clin Otolaryngol 1998;23:462466.

$14 \mathrm{Wu} \mathrm{CH}$, Murofushi T: The effect of repetition rate on vestibular evoked myogenic potential. Acta Otolaryngol 1999;119:29-32. 